\title{
Integrierte Versorgung unter gemeindepsychiatrischer Steuerung - Kontra
}

\author{
Integrated Health Care Managed by Community Psychiatric Services - Contra
}

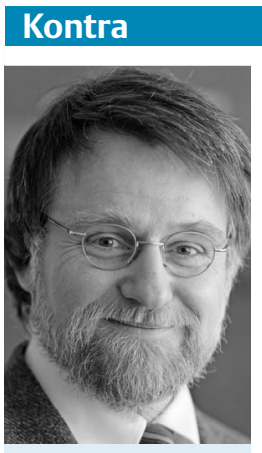

Thomas Schillen
Die Psychiatriereform in Deutschland ist vor vielen Jahren stehen geblieben. Wir leisten uns noch immer eine Zersplitterung der Versorgung über ambulante und stationäre Behandlungssektoren und diverse Sozialgesetzbücher hinweg, die zu schlechteren Behandlungsergebnissen und höheren Kosten führt. Die nachgewiesene Wirksamkeit einer sektorübergreifenden Versorgung [1 -4] wird in der Weiterentwicklung der psychiatrischen Versorgung nicht aufgegriffen, obwohl eine evidenzbasierte integrierte Versorgung zwingend erforderlich ist.

Eine Klinik mit sozialpsychiatrischer Ausrichtung ist nach meinem Verständnis immer noch integraler Bestandteil eines gemeindepsychiatrischen Verbunds (GPV). Wenn eine solche Klinik Projekte zur Integration der Versorgung und zu Assertive Community Treatment (ACT) $[2,3,5,6]$ voranbringt, dann kann ich aufgrund der positiven Erfahrungen mit unserem Hanauer Modell nur Argumente zum Pro des Debattentitels anführen.

Fokussieren wir aber um der Kontroverse willen auf Projekte zur Integrierten Versorgung durch außerklinische Partner im GPV, dann werden am Beispiel des Netzwerkes Psychische Gesundheit (NwPG) erhebliche Probleme sichtbar:

Beim NwPG-Vertrag erhält der Leistungserbringer für jeden eingeschriebenen Patienten eine jährlich degressive Kopfpauschale. Die Höhe der Pauschale hängt von dem erwarteten Risiko einer Klinikbehandlung ab, das die Krankenkasse anhand der Patientenmerkmale prognostiziert. Für Jahrespauschalen zwischen etwa 500 und $2000 €$ übernimmt der Leistungserbringer die Kosten einer Krankenhausbehandlung, falls diese trotz seiner Interventionen nicht vermeidbar ist. In der ursprünglichen Konzeption des Vertrages durch die Techniker Krankenkasse gingen auch Behandlungen der Institutsambulanz einer Klinik zulasten des IVLeistungserbringers. In den aktuellen Verträgen wurden die finanziellen Risiken für den IV-Vertragsnehmer, soweit dies öffentlich ist, nach Höhe der Jahrespauschalen und Krankenhauskosten leicht entschärft. Dennoch bleibt der irritierende Eindruck, dass der Vertrag primär entlang der wirtschaftlichen Interessen der Krankenkasse zur Begrenzung von Krankenhauskosten konzipiert ist. Für diesen Eindruck spricht auch, dass dieser IV-Vertrag in vielen Regionen ohne die Beteiligung der Krankenhäuser geschlossen wurde. Der Vollständigkeit halber sei angemerkt, dass diesen IV-Verträgen mit der fehlenden Integration ambulanter und stationärer Leistungen zusätzlich auch die rechtlichen Voraussetzungen nach §140a SGB V fehlen.

Mit dem NwPG-Vertrag kommt der Leistungserbringer in die Zwangslage, Patienten mit stationärem Behandlungsbedarf gegen seine eigenen wirtschaftlichen Interessen ins Krankenhaus einweisen oder die medizinischen Risiken einer unterlassenen Einweisung tragen zu müssen. Hinweise, dass gerade die schwer psychisch Kranken möglichst nicht in den IV-Vertrag eingeschrieben oder auch wieder ausgesteuert werden, um die wirtschaftlichen Risiken zu begrenzen, überraschen dabei nicht.

Die konkrete Umsetzung des NwPG-Vertrags in über 10 Regionen in Deutschland scheint regional mit sehr unterschiedlicher Qualität zu gelingen. Die Qualität der Umsetzung leidet insbesondere, wenn sich beispielsweise die erforderlichen ärztlichen Ressourcen in einer Region nicht finden lassen.

Das sehr gut evaluierte Hamburger Modell erreicht mit seiner ACT-Versorgung für besonders schwer psychisch Kranke ein besseres Funktionsniveau, eine bessere Lebensqualität, niedrige Drop-out-Raten, aber keine Kosteneinsparung gegenüber der stationären Krankenhausbehandlung [3]. Vor diesem Hintergrund stellen sich die unzureichenden Kopfpauschalen und die hohen wirtschaftlichen Risiken für den NwPG-Leistungserbringer als die zentralen konzeptionellen Fehler dar.

Nehmen wir an, diese Fehler ließen sich durch Anpassungen des Vertrags lösen und betrachten wir dann das Team zur stationsäquivalenten ambulanten Akutbehandlung (AAB) im Hanauer Modell [6]: Dieses Team in Stationsstärke behandelt solche Patienten ambulant, deren aktueller Schweregrad der Erkrankung im bisherigen Versorgungssystem eine stationäre Krankenhausbehandlung erfordert hätte. Dabei steht das gesamte Behandlungsangebot einer stationären Versorgung zur Verfügung. Darüber hinaus bietet dieses Team bei Bedarf HomeTreatment und stellt ein Kriseninterventionsteam. Patienten mit schweren rezidivierenden und chronischen Störungen werden langfristig begleitet. Der Fokus liegt auf der Autonomie und den Ressourcen des Patienten. Die Patienten kommen freiwillig, Zwangsmaßnahmen sind nicht möglich, aggressive Situationen treten kaum auf.

Das AAB-Team ist sehr erfolgreich darin, zusammen mit dem Patienten und seinem sozialen System Wege zu finden, die Akutbehandlungen als Krankenhausleistung ohne stationäres Bett gelingen lassen. Würde man dieses AAB-Team aus der Klinik in eine NwPG-Struktur ausgliedern, dann würde dies zweifelsohne die Krankenhaustage unverändert minimieren. Trotz der hohen fachlichen und personellen Ressourcen des AAB-Teams blieben aber etwa $60 \%$ der stationären Leistungen unvermeidbar.

Die bisherigen Ergebnisse im Hanauer Modell zeigen, dass etwa $40 \%$ des stationären Budgets in den nächsten Jahren in ambulante Leistungen überführt werden können. Übertragen auf die psychiatrischen Krankenhauskosten in Deutschland könnten damit ohne Mehrkosten etwa 45 Milliarden $€$ für eine Ambulantisierung 
der Psychiatrie bereitgestellt werden. Damit ließen sich die fehlenden Versorgungselemente wie beispielsweise ACT, Home-Treatment und ambulante Kriseninterventionsteams realisieren. Die Budgets typischer IV-Verträge erreichen dagegen vielleicht $1-2 \%$ dieses Finanzvolumens.

Damit Ansätze wie der NwPG-Vertrag funktionieren und relevante strukturelle Veränderungen herbeiführen könnten, wäre eine substanziell höhere Finanzierung dieser Verträge erforderlich. Damit würden diese Ansätze aber in gleichem Maße zu einer zusätzlichen Belastung des SGB-V-Budgets im Gesundheitssystem mit Fehlanreizen zum Aufbau unnötiger Doppelstrukturen. Für eine nachhaltige Verbesserung im Sinne der PsychiatrieEnquête brauchen wir vielmehr eine Reform der Kliniken, die bestehende Budgets ohne Mehrkosten flexibilisiert.

Letztlich geht es um die Frage, welche Versorgungsqualität wir für welchen Schweregrad an psychischer Erkrankung bereitstellen wollen. Diese Frage bedarf eines gesellschaftlichen Diskurses und kann nicht den Partikularinteressen von Kostenträgern und Klinikkonzernen überlassen bleiben. Das Krankenhaus ist nicht Selbstzweck, genauso wenig wie der GPV. Integrierte Versorgung entsteht aber nicht einfach dadurch, dass eine Krankenkasse aus betriebswirtschaftlichem Kalkül gemeindepsychiatrischen Leistungserbringern Zugang zu neuen Erlösquellen im
SGB V verschafft. Zur Verbesserung der Versorgung und Integration im GPV gehört vielmehr, dass die außerklinischen Leistungserbringer in gleicher Weise wie die Kliniken eine Pflichtversorgung für ihr Einzugsgebiet übernehmen. Dazu gehört ferner, dass Sozialhilfeträger ausreichende Ressourcen bereitstellen, damit ein Wohnheimbedarf des Patienten nicht mehr gleichbedeutend ist mit jahrelanger Wartezeit oder Verlegung irgendwohin in Deutschland. Die „Steuerung“ in einem solchen Verbund läge beim Patienten, der im Rahmen der Hilfeplanung seine tragenden sozialen und therapeutischen Beziehungen deutlich macht. Diese wären dann individuell verschieden beim außerklinischen Leistungserbringer, beim niedergelassenen Arzt oder auch in einer Institutsambulanz und einem ACT-Team des Krankenhauses zu finden.

Integrierte Versorgungsverträge unter gemeindepsychiatrischer Steuerung nach Art des NwPG-Vertrags beseitigen nicht die Zersplitterung der psychiatrischen Versorgung. Sie belasten das Gesundheitssystem vielmehr mit zusätzlichen Kosten und Doppelstrukturen. Eine Flexibilisierung und Ambulantisierung der Krankenhausleistungen wäre dagegen endlich wieder eine grundlegende Perspektive im

Sie haben eine eigene Meinung zu diesem Thema? Dann schreiben Sie uns an: psychiat-praxis@thieme.de!
Sinne der Psychiatrieenquete für psychisch erkrankte Menschen.

\section{Literatur}

1 Roick C et al. Das Regionale Psychiatriebudget: Kosten und Effekte eines neuen sektorübergreifenden Finanzierungsmodells für die psychiatrische Versorgung. Psychiat Praxis 2008; 35: 279-285

2 Deister $A$ et al. Veränderung der psychiatrischen Versorgung durch ein Regionales Budget. Psychiat Prax 2010; 37: 335-342

3 Lambert $M$ et al. Integrierte Versorgung von Patienten mit psychotischen Erkrankungen nach dem Hamburger Modell: Teil 1. Psychiat Prax 2014 (im Druck)

4 Karow $A$ et al. Integrierte Versorgung von Patienten mit psychotischen Erkrankungen nach dem Hamburger Modell: Teil 2. Psychiat Prax 2014 (im Druck)

5 Schillen T, Thiex-Kreye M. Ambulante Behandlung durch die psychiatrische Klinik G+S 2012; 4: 53-59

6 Schillen T, Thiex-Kreye M. Hanauer Modell Perspektiven für die psychiatrische Versorgung in Deutschland. Kerbe 2014; 2: 11 - 15

Korrespondenzadresse

Priv.-Doz. Dr. med. Dipl. Phys. Thomas Schillen

Klinikum Hanau $\mathrm{GmbH}$,

Klinik für Psychiatrie und Psychotherapie

Julius-Leber-Straße 2a

63450 Hanau

thomas_schillen@klinikum-hanau.de

Bibliografie

Dol http://dx.doi.org/

10.1055/s-0034-1369954

Psychiat Prax 2014; 41: 183-184

(c) Georg Thieme Verlag KG

Stuttgart · New York

ISSN 0303-4259 